\title{
Oral Communications
}

\author{
(OR 01 to OR 24)
}

\author{
Anti-prostasome autoantibodies in serum from \\ prostate cancer patients \\ K.G. RONQUIST, L. CARLSSON, G. RONQUIST, \\ L. LARSSON \\ Department of Medical Sciences, Sweden \\ Email : goran.ronquist@medsci.uu.se
}

$\underline{\text { OR } 01}$

Objectives : Prostasomes are secretory granules synthesized, stored and released by normal and neoplastic human prostate epithelial cells. The average size is $150 \mathrm{~nm}$ in diameter (range 50-500 nm) and the bilayered membrane has a very high cholesterol/phospholipid ratio making up an interesting new biological environment. Prostasomes seem to have several important functions in the reproduction process. Prostasomes escaping from the urogenital tract mount an immune response against themselves. Other cell types have also been proved to produce extracellular vesicles in a similar way called exosomes. Each vesicle type has a unique membrane protein composition reflecting the cell from which it originates. The aim with this study was to identify the prostasome-derived proteins that were immunogenic in serum from prostate cancer patients.

Materials and Methods : The aim was to identify the prostasome-derived proteins that were immunogenic in prostate cancer patients. Prostate cancer patients' sera (n $=44$ ) with high enzyme-linked immunosorbent assay (ELISA) titers against prostasomes were selected for immunoblotting against purified seminal prostasomes separated on 2D gels. Twenty-five of the recognized proteins were isolated and analyzed by means of mass spectrometry.
Results : Out of 44 patients' sera, $31(70 \%)$ demonstrated in immunoblotting experiments reactivity against several prostasomal protein bands in the molecular weight range of $10-200 \mathrm{kDa}$. Some of the bands $(55,70$ and $170 \mathrm{kDa})$ were more frequently recognized by the patients' sera. Concomitantly run control sera generated only very weak or no bands at all. The most frequently occurring prostasomal proteins were identified as heat shock proteins (HSP 70 , 71) and clusterin.

Conclusion : Prostate cancer consistently remains a difficult clinical enigma. Therefore, the development of novel strategies for diagnosis and treatment (e.g. immunotherapy) of prostate cancer is essential. This study identified the most important molecular targets of autoantibodies against prostasomes generated in connection with the development of prostate cancer in man. These immunogenic prostasomal proteins could be appropriate target molecules for specific immunotherapy of prostate cancer patients.

$\underline{\mathrm{OR} 02}$

Prospective multicenter study on safety and efficacy of intramuscular injections of $1000 \mathrm{mg}$ testosterone undecanoate in hypogonadal men under conditions resembling real-life

\section{H.M. BEHRE1, J. ELLIESEN2, AND THE TESTOSTERONE UNDECANOATE STUDY GROUP}

1 Andrology Unit, Dept. of Urology, Martin-LutherUniversity, Halle, Germany ; 2 Schering AG, Berlin, Germany

Objective : The study was performed to evaluate intramuscular testosterone undecanoate (TU) for treatment 
of hypogonadal men under conditions which resemble reallife situations as closely as possible (e.g., variable injection intervals, minimum selection of patients, i.e., including hypogonadal patients with co-morbidities). It was intended to test the safety profile of TU under long-term treatment with respect to serum levels of prostate-specific antigen (PSA) [primary study variable], prostate size measured by transrectal ultrasonography, physical examinations and standard safety laboratory. A further aim was to demonstrate that sustained serum testosterone $(T)$ levels in the physiological range can be retained under real-life conditions, preserving satisfaction with the treatment.

Design : Open-label, prospective, multicenter (16 centers in Germany), one-arm study.

Material and Methods : Men with hypogonadism, aged 18 - 75 years, with serum total T levels $<10 \mathrm{nmol} / \mathrm{l}$ and no contraindications for $\mathrm{T}$ therapy were included. $1000 \mathrm{mg}$ TU (Nebido®) were given at individually adjustable loading intervals of $6-10$ weeks between the first 2 injections and maintaining intervals of $10-14$ weeks (total: 6 TU injections).

Results : Of 161 patients screened, 96 could be included (age, $48.6 \pm 12.7$ years [mean \pm SD]; height, $178.85 \pm 7.84 \mathrm{~cm}$; body weight, $93.33 \pm 15.71 \mathrm{~kg}$; body mass index, $29.22 \pm 4.80$ $\mathrm{kg} / \mathrm{m}^{2}$ ). Trough concentrations of total $T$ (considered as an indicator of the adequacy of substitution) increased from 5.60 $\pm 3.03 \mathrm{nmol} / /$ at baseline to levels between $9.79 \pm 3.96$ and $14.02 \pm 6.06 \mathrm{nmol} / \mathrm{l}$ in the course of treatment phase. Median values of trough $T$ after the $2^{\text {nd }}$ TU injection were $12-13.25$ $\mathrm{nmol} / \mathrm{l}$. Mean PSA serum concentration of $0.81 \pm 0.80 \mathrm{ng} / \mathrm{ml}$ at screening moderately increased to $1.23 \pm 1.33 \mathrm{ng} / \mathrm{ml}$ after the 6 th TU injection. At the time of the 5th TU injection, $75 \%$ of the patients had PSA values $\leq 1.40 \mathrm{ng} / \mathrm{ml}$ (3rd quartile); at the time of the final examination the 3rd quartile was $1.5 \mathrm{ng} / \mathrm{ml}$. There were 7 patients with elevated PSA values above the age-specific normal range at the final examination compared to 1 patient at screening; however, only 2 of them had values within the age-specific alert ranges. Malignant changes were excluded by prostate biopsies in 4 patients with a PSA level above $4 \mathrm{ng} / \mathrm{ml}$. Mean prostate volume increased from $20.66 \pm 11.44 \mathrm{ml}$ at screening to $22.54 \pm 13.11$ $\mathrm{ml}$ at the time of the 5 th injection and $22.63 \pm 12.84 \mathrm{ml}$ at the final examination. Physical examinations and standard safety laboratory confirmed the safety of TU. $92.5 \%$ of the patients assessed the treatment with TU as satisfying. Of them $40.4 \%$ were "very much satisfied" and $43.6 \%$ "much satisfied".

Conclusions : Under conditions resembling real-life situations, intramuscular TU (Nebido®) appears to be a safe and efficient drug for treatment of male hypogonadism. The majority of patients express high satisfaction with the treatment.

Support : The study was supported by Schering AG, Germany.
Androgens modulate the availability of active glucocorticoids by regulating 11-SS-HSD1 activity in murine adipocytes in vitro

\section{A.M. ISIDORI, S. PIEROTTI, D. GIANFRILLI, E. GIANNETTA, V. BONIFACIO AND A. LENZI}

Cattedra di Endocrinologia, Dipt. Di Fisiopatologia Medica, Univ. "La Sapienza" di Roma.

Objective : In humans, testosterone treatment affects body fat distribution and metabolism. The mechanisms involved in these processes are not yet entirely understood. We hypothesized that sex hormones modulate the activity of [type 1] 11-ß-hydroxy-steroid-dehydrogenase (11-HSD1) -the enzyme involved in the intracellular activation of glucocorticoids.

Design : We measured intracellular fat accumulation, leptin production (Lep) and 11-HSD1 activity in differentiating or fully mature $3 T 3-L 1$ cells under the conditioning effects of sex hormones.

Materials and Methods : 11-HSD1 activity was estimated calculating the molar conversion of [H3]-dehydrocorticosterone (DHC) into [H3]-corticosterone. Steroids were extracted using solvents, separated by TLC and measured by B-scanning of the TLC plate. Testosterone (T), DHT and estradiol (E2), in the presence/absence of androgen receptor antagonist (flutamide) and aromatase inhibitor (formestane) were tested at various time-points (2 to 6 days) and doses ( $1 \mathrm{nmol}$ to 1 $\mu \mathrm{mol} / \mathrm{L}$ ) using carchoal-stripped serum and/or minimum serumfree cell cultures. Various treatment condition using a synthetic Liver X Receptor (LXR) Agonist were also tested.

Results : We found that 3T3-L1 cells acquire 11-HSD1 activity at early stages of differentiation and that the activity increases steadily during differentiation in parallel with fat accumulation. We demonstrated that androgens ( $T$ and DHT) enhance 11HSD1 activity dose-dependently either in mature and differentiating cells (form $10 \%$ when treated with $1 \mathrm{nM} \mathrm{T}$, to $40 \%$ with $1 \mu \mathrm{M} \mathrm{T}$; conversion measured at 90 minutes incubation with $15 \mathrm{nM}$ [H3]-DHC + $15 \mathrm{nM}$ cold-DHC). We also showed that androgen modulation of enzymatic activity was similar to that achieved by insulin $(5 \mathrm{ng} / \mathrm{ml})$-a known regulator of 11-HSD1. Conversely, LXR agonist inhibited 11HSD1 activity dose-dependently. We also found that the effect of androgens, but not insulin, were partially blunted by pretreatment with flutamide $(200 \mathrm{nM})$. In addition, we showed that $T$ treatment on differentiating cells, inhibited leptin production and fat accumulation/release while increasing 11-HSD1 activity. The latter finding suggests that the effects of androgens on the enzyme were independent of their effects on differentiation and fat storage. 
Conclusions : In summary, we showed that in an experimental murine model androgens may regulate adipocytes metabolism by affecting the availability of intracellular glucocorticoids. The implication of these findings on testosterone replacement therapy remains to be established with human models and in vivo studies.

$\underline{\mathrm{OR} 04}$

\section{Sex steroid regulation of kisseptin/GPR54 system, a new regulator of the neuroendocrine reproductive axis, in human fetal GnRH secreting neurons}

\author{
A. MORELLI1, R. MANCINA1, L. VIGNOZZI1, \\ M. LUCONI2, G. FORTI1, M. MAGGI1 \\ 1 Andrology and 2 Endocrinology Units, Department of \\ Clinical Physiopathology ; 3 Department of Anatomy, \\ Histology and Forensic Medicine, University of Florence, \\ Florence, Italy (a.morelli@dfc.unifi.it)
}

Objective : The hypothalamic $\mathrm{GnRH}$ neurons, arising from the olfactory placode region, remain immature until puberty. The molecular mechanisms underlying their reawakening at puberty remain to be completely elucidated. Recently, the G protein-coupled receptor 54 (GPR54) and its endogenous ligand kisspeptin, encoded by the KISS-1 gene, have been crucially involved in the hypothalamic mechanisms that time puberty. We used the previously characterized human fetal olfactory $\mathrm{GnRH}$-secreting neurons, FNC-B4, in order to study the KISS-1/GPR54 system.

Design and Methods : FNC-B4 are immature neurons that can differentiate in vitro and express both neural and olfactory markers. Moreover, these cells express and release $\mathrm{GnRH}$ in response to sex steroids and odorants, as well as migrate in response to $\mathrm{GnRH}$ and endothelin-1. By real-time RT-PCR and western blot analysis we studied the kisspeptin and GPR54 expression in FNC-B4 in response to sex steroids stimulation.

Results : We report that these human neuronal precursor cells express both gene and protein of kisspeptin and GPR54. With immunohistochemistry we localized both kisspeptin and GPR54 in sections of fetal olfactory mucosa, as well as in FNCB4 cells. Moreover, we observed that sex steroids regulated both gene and protein expression of KISS-1/GPR54 system. In particular, we found that increasing concentrations $(0.01$ $100 \mathrm{nM})$ of 17 beta-estradiol significantly decreased $(I C 50=0.3 n M)$ in a dose-dependent manner the amounts of KISS-1 and GPR54 mRNA. Conversely, androgens (DHT,
$0.01-100 \mathrm{nM}$ ) stimulated gene expression of both KISS-1 and GPR54. In addition, we found that leptin ( $1 \mathrm{nM}$ ), an adypocytederived hormone known to act on the hypothalamus to influence reproduction, increased the expression of KISS-1 and GPR54 mRNAs.

Conclusions : Overall, our results indicate that FNCB4 cells represent a valuable tool to study in vitro the regulation of KISS1/GPR54 system in order to identify those permissive signals that determine the precise timing of puberty onset and related disorders.

$\underline{\text { OR } 05}$

Inhibition of Phosphodiesterase type 5 is
associated to an in vivo and in vitro improved
activity of circulating angiogenic cells in men
with erectile dysfunction

\section{S. FRANCAVILLA1, M. BOCCHIO1, F. PELLICCIONE1,} G. PASSAQUALE 2 , S. NECOZIONE 3 , C. FERRI 2

Chairs of 1 Andrology, 2 Internal Medicine, 3 Epidemiology, University of L'Aquila, Italy. Presenting author e-mail : HYPERLINK "mailto : sandrof@univaq.it" sandrof@univaq.it

Objective : Circulating angiogenic cells (CACs) contribute to repair of the vessel wall and dysfunctional CACs are associated to endothelial dysfunction in men with vascular risk factors (VRFs).

Design : We investigated whether inhibition of phosphodiesterase type 5 (PDE5) in men with erectile dysfunction (ED) and VRFs is accompanied to changes of activity of CACs.

Materials and Methods: Thirty six men with ED and VRFs were randomised to 4 weeks of tadalafil ( $20 \mathrm{mg} /$ other day) or placebo treatment. The number of ex vivo expanded functional CACs, identified by uptake of 1,1'-dioctadecyl-3, 3,3', 3'tetramethylindocarbocyanine-labelled acetylated low-density lipoprotein (DiLDL) and concomitant Ulex europaeus agglutinin I (UEA-1) binding, was determined at baseline and after treatment.

Results : The number of cells double-positive for DiLDL and for UEA-1, per high-power field fluorescence microscopy was significantly reduced in patients compared to 10 controls $(26.88 \pm 6.3$ and $74.41 \pm 17.1$ respectively; $P<0.0001)$ and was markedly increased after tadalafil treatment $(40.69 \pm 13.07$ versus $25.82 \pm 6.49 ; \mathrm{P}<0.0001)$ but not after placebo. The percentage variation of the number of CACs was correlated 
with the percent change of Flow-mediated dilation in the brachial artery by ultrasound, after treatment ( $R=0.59$, $\mathrm{P}=0.0004)$. The migratory activity of ex vivo expanded CACs determined in a Boyden chamber system, was significantly increased after incubation for 3 hours with tadalafil (10-6M) (number of migrated cells: $1073 \pm 363$ versus $702 \pm 204$; $P=0.0007$ ). The effect on number of cells after tadalafil incubation was not due to an increased proliferation of CACs. CACs proliferation was assessed by the incorporation into cellular DNA of 5-bromo-2'-deoxyuridine (BrdU) and subsequent staining with a TRITC-labelled mouse monoclonal antibody anti-BrdU. The number of BrdU-positive cells evaluated with an inverted fluorescence microscope did not change after 3 and 24 hours of tadalafil incubation (10-6M).

Conclusion : In conclusion PDE5 inhibition has a beneficial in vivo and in vitro effect on vascular homeostasis by improving circulating angiogenic cell function.

$\underline{\text { OR } 06}$

\section{Testosterone regulates RhoA/Rho-kinase signalling in two distinct animal models of chemical diabetes}

\author{
L. VIGNOZZI1, A. MORELLI1, S. FILIPPI2, M. LUCONI3, \\ G. FORTI1, M. MAGGI1 \\ 1 Andrology and 3 Endocrinology Units, Dept. of Clinical \\ Physiopathology ; 2 Interdep. Lab of Functional and \\ Cellular Pharmacology of Reproduction, Depts. of \\ Pharmacology and Clinical Physiopathology; University of \\ Florence, Florence, Italy (I.vignozzi@dfc.unifi.it)
}

Objectives : The RhoA/Rho-kinase (ROK) signalling pathway is up-regulated in erectile tissue of diabetes animal models and may contribute to diabetes-related erectile dysfunction (ED). In previous studies we demonstrated that testosterone (T) restores diabetes-induced ED by influencing the NO/cGMP/PDE5 pathway. The aim of our study was to investigate the effect of T on RhoA/ROK signalling in course of diabetes.

Design and Methods : Two distinct animal models of chemical diabetes: alloxan-induced in the rabbit and streptozotocininduced in the rat were used.

Results : In both models, hypogonadism was observed, characterized by reduced $\mathrm{T}$ and atrophy of androgendependent accessory glands. Diabetic animals showed a significant increase in responsiveness to increasing concentrations of Y27632, a highly selective ROK inhibitor, as evaluated either by "in vitro" contractility study (diabetic rabbit) and "in vivo" as erectile response elicited by electrical stimulation of cavernous nerve (diabetic rats). T-substitution (30 mg/kg, weekly) completely reverted hypogonadism and diabetes-induced penile hypersensitivity to Y27632. To test whether this effect was due to a T-dependent regulation of RhoA/ROK gene expression, we measured RhoA/ROK mRNA. Both isoforms of ROK (ROK1 and ROK2) were analyzed by real time RT-PCR in penile samples of each rat group. We found that ROK1 mRNA was significant increased $(p<0.05)$ in penile tissues from diabetic animals and restored to the control values by $T$, as also confirmed by semiquantitative RT-PCR in rabbit. Conversely, expression of RhoA and ROK2 mRNAs was not influenced neither by diabetic condition and by $\mathrm{T}$ administration. Accordingly, ROK1 protein expression, as evaluated by western blot and immunohistochemistry analysis, resulted increased in penile samples from diabetic animals and normalized by $\mathrm{T}$.

Conclusions : Our data further support the hypothesis that the activation of RhoA/ROK signalling contributes to diabetesrelated erectile dysfunction. Moreover, treating hypogonadism in course of diabetes, may restore erectile function by maintaining a correct balance between pro-erectile NO/cGMP and anti-erectile RhoA/ ROK pathways.

$\underline{\text { OR } 07}$

Cryptorchidism and in utero exposure to
endocrine disruptors in Nice area : absence of
correlation between neonatal examination and
concentrations of selected xenobiotics in cord
blood and maternal milk

F. BRUCKER-DAVIS1-2, A. BONGAIN 3, B. DUCOT4, P. THONNEAU5, K. WAGNER-MAHLER6, P. FÉNICHEL1-2

1 Endocrinology Department, CHU Nice, 06200 Nice, France. 2 Unité INSERM, Nice, France. 3 Gynaecology Department, CHU Nice, 06200 Nice, France. 4 Unité INSERM, Paris, France. 5 CHU Toulouse, France. 6 Pediatry Department, $\mathrm{CHU}$ Nice, Nice France Corresponding author : brucker-davis.f@chu-nice.fr

The etiology of cryptorchidism, a frequent congenital malformation, is not completely elucidated, involving genetic and acquired factors.

Objectives: We decided to test the hypothesis that environmental xenobiotics with hormonal action (endocrine disruptors) are involved. Data on in utero exposure are scarce in France. Thus, we realized a snapshot of in utero exposure 
Design : We have studied over 3 years (2002-2005) newborn boys born at 2 maternity wards (CHU Nice, and CHG Grasse), screening them for cryptorchidism and for exposure (cord blood and maternal milk) to selected xenobiotics known for their anti androgenic and/or estrogenic effects. In addition, parents were asked to fill confidential questionnaires about their reproductive history, their origin, occupation, hobbies and other potential exposure. This study was funded as a PHRC 2001 and apporved by the Ethical board of our institutions.

Material and methods : Of 6246 boys born at or after 34 weeks, 101 were cryptorchid (incidence of $1.6 \%$ in Nice and Grasse). 95 were included in this study ( 48 from Nice and 47 from Grasse), as well as 2 controls matched for gestationnal age, date and place of birth, and when possible ethnic origin. 151 serum ( 67 cryptorchid and 84 controls) and 125 milk (56 cryptorchid and 69 controls) were tested for the presence of 7 non planar PCBs, DDE, dibutylphtalate and its metabolite monobutylphtalate, hexachlorobenzene, linuron, procymidone and vinclozolin. Xenobiotic measurements were performed by gaz chromatography coupled to mass spectometry at accredited laboratory (Laboratoire de l'Environnement de l'Agglomération Niçoise).

Results : All samples were positive for one or more xenobiotics confirming the ubiquitous exposure of foetuses/infants in our area. Concentrations were generally higher in milk, as expected for lipophilic compounds. Concentrations, however, were often lower than in other studies. We established scores of exposure (high, low, negligible) using composite scores including ÂPCBs, DDE and phthalate. We have found no significant differences between cryptorchid and controls. As predictive factor of high exposure, we found maternal age, and for DDE, the geographical origin of the mother (subsaharian and north Africa)

Conclusion : The incidence of cryptorchidism in our area is $1.6 \%$ at birth. The exposure to endocrine disruptors is widespread, but at a lower level than in other reported series. Our current data does not support a direct link between exposure to a given xenobiotic and cryptorchidism in our population.

\section{Does histopathological tumor type or vascular invasion influence spermatogenesis in testicular cancer?}

\author{
J.E. LACKNERa, A. KOLLERb, G. SCHATZLa, \\ M. MARBERGERa, C. KRATZIK a \\ Medical University of Vienna, Vienna, Austria \\ a Department of Urology \\ b Department of Pathology
}

Objectives : To assess quality and activity of spermatogenesis in the contralateral testicle at the time of orchiectomy and to assess whether any tumor related factor such as tumor type or vascular invasion is a risk factor for impaired spermatogenesis. An additional aim was to investigate if areas with normal spermatogenesis for a possible harvesting of sperms for artificial reproductive technologies could be found in the contralateral testicle.

Methods : Contralateral biopsy specimens at the time of unilateral orchiectomy from patients with testicular cancer were evaluated using the Johnsen score. Median and highest detectable Johnsen score were counted. The Johnsen score was correlated to histopathological tumor type such as seminoma and non seminomatous germ cell tumor (NSGCT) and to TNM stage.

Results : From overall 76 patients 36 had a seminoma, while 40 patients had a NSGCT. In all patients a stage I disease was diagnosed: in the seminoma group 13 pT1 and 23 pT2 tumors and in the NSGCT group 9 pT1, 29 pT2 and 2 pT3 tumors. The median Johnsen score of 8.9 in seminomas did not differ significant to that in NSGCT $8.6(p=0.348)$. According to the presence of vascular invasion similar results were seen with a median Johnsen score of 8.8 (8.1-9.2) in pT1 and $8.8(8.2-$ $9.5)$ in $p T 2$ or higher stages $(p=0.389)$. Areas with a highest Johnsen score above 8 were found in $88.9 \%$ in seminomas and $92.5 \%$ in NSGCT.

Conclusion : Testicular cancer is associated with impaired spermatogenesis. Neither the histopathological tumor type nor the presence of vascular invasion was correlated to significant reduced spermatogenesis. When testicular sperm extraction from contralateral testis at the time of orchiectomy is planned, there is a high chance to find areas with normal spermatogenesis. 
and $\mathrm{T} 60$ (OR 4.4, 95\% $\mathrm{Cl} 1.2-16$ ). Those hypogonadal at $\mathrm{T0}$ were at higher risk of hypogonadism at T6 (OR $53,95 \% \mathrm{Cl}$

Risk factors for development of hypogonadism in men treated for testicular cancer: a longitudinal study

\section{J. EBERHARD2, 0. STARL1-2, Y. GIWERCMAN2, E.C. SALMONSON 3 , A. RIGNELL-HYDBOM4,}

\section{A. GIWERCMAN2.}

Department of Oncology, Lund University Hospital, Lund University, Lund, Sweden.

Fertility Centre, Scanian Andrology Centre, Malmö

University Hospital, Lund University, Malmö, Sweden.

Department of Radiology, Lund University Hospital, Lund University, Lund, Sweden.

Department of Environmental and Occupational Medicine, Lund University Hospital, Lund University, Lund, Sweden.

Background : TGCC (Testicular Germ Cell Cancer) patients have been suggested to be at risk of developing reduced Leydig cell function and hypogonadism because of both the disease per se and its treatment. The aim of current study was to define clinical and laboratory risk factors for developing hypogonadism in TGCC patients.

Patients and Methods : During the period 2001-2005, 143 consecutive TGCC patients followed at the oncological outpatient clinic, Lund University Hospital, were enrolled in the study. Blood samples were collected at fixed time points (after orchidectomy, prior to further therapy [T0] and 6, 12, 24, 36 and 60 months [T6, T12, T24, T36, T60] after completion of therapy). Hypogonadism was defined as serum testosterone $<10 \mathrm{nmol} / \mathrm{L}$ and/or $\mathrm{LH} \geq 10 \mathrm{IU} / \mathrm{L}$. Patients having increased hCG levels post-orchidectomy were not included. As possible predictive factors the following were tested: hypogonadism at TO (yes/no); age at diagnosis $(<30 / \geq 30)$; type of treatment (adjuvant chemotherapy [ACT]/ 3-4 cycles chemotherapy [HCT]/adjuvant radiotherapy [RT]); stage of disease (I/II/III or IV); contralateral testicular microlithiasis on ultrasound (yes/no); testicular consistency (normal/abnormal); testicular volume $(<15 \mathrm{ml} / \geq 15 \mathrm{ml})$; androgen receptor CAG and GGN repeat length. For each post-treatment time point, odds ratios for hypogonadism were calculated by use of binary logistic regression. As controls 104 Swedish fishermen aged 24-49 years were used (median: 40 years).

Results : As compared to controls, significantly increased odds ratios (OR) for hypogonadism was found at T6 (OR: $5.3,95 \%$ confidence interval $[\mathrm{Cl}]$ 1.6-17) and T12 (OR 3.2, $95 \% \mathrm{Cl} 1.1-9.3)$ in HCT treated men. Patients treated with ACT or RT did not have any higher risk of becoming hypogonadal than controls. Microlithiasis was predictive for hypogonadism at T0 (OR 11, 95\% Cl 1.2-112), T12 (OR 3.9, 95\% Cl 1.1-13), T24 (OR 3.0, 95\% Cl 1.0-8.8), T36 (OR 5.4, 95\% Cl 1.7-17)
19-145), T12 (OR 125, 95\% Cl 37-430), T24 (OR 88, 95\% Cl 26-300) and T36 (OR 121, 95\% $\mathrm{Cl} 32-460$ ). We did not find any predictive value of age, stage of disease, AR polymorphisms, volume or consistency of contralateral testicle.

Conclusion : Hypogonadism at T0 together with type of treatment and microlithiasis of the contralateral testis seem to be predictive factors for post-treatment hypogonadism in men treated for TGCC. These risk factors should be taken into consideration in the clinical follow-up of men cured for testicular cancer.

$\underline{\mathrm{OR} 10}$

\author{
Increase in testicular cancer incidence in the \\ Midi-Pyrenees region, France
}

M. WALSCHAERTS1-4, E. HUYGHE1-2, R. MIEUSSET1, M. DAUDIN1-3, P. PLANTE1-2, P. THONNEAU1-2

1 Equipe de Recherche en Fertilité Humaine (EA 3694), Hôpital Paule de Viguier, 31059 Toulouse

2 Service d'Urologie et d'Andrologie, Hôpital Paule de Viguier, 31059 Toulouse

3 CECOS (Centre de Conservation du Sperme), Hôpital

Paule de Viguier, 31059 Toulouse

4 Corresponding author: Hôpital Paule de Viguier, Groupe de Recherche en Fertilité Humaine (EA 3694), 330 av de Grande Bretagne, TSA 70034, 31059 Toulouse Cedex 9, France.walschaerts.m@chu-toulouse.fr

Numerous international studies have observed constant and recent progression in the incidence rate of testicular cancer since the middle of the 20th century in industrialised countries.

Objective : To find a predictive mathematical model of the time trend of testicular cancer incidence in the Midi-Pyrenees region of France.

Design - Materials and Methods : Study of a consecutive series of 506 cases of testicular cancer occurring over a 20 year period (1980-1999) in the Midi-Pyrenees region. Patients were recruited in a standardised manner by the CECOS and the principal anti-cancer centres of the region, which made it possible to obtain a quasi-representative study population of testicular cancers in this area. Date of birth, year of tumour diagnosis and histological type were available for all patients. Incidence rates were standardised to the world population. An age-period-cohort model was adjusted using Poisson regression. 


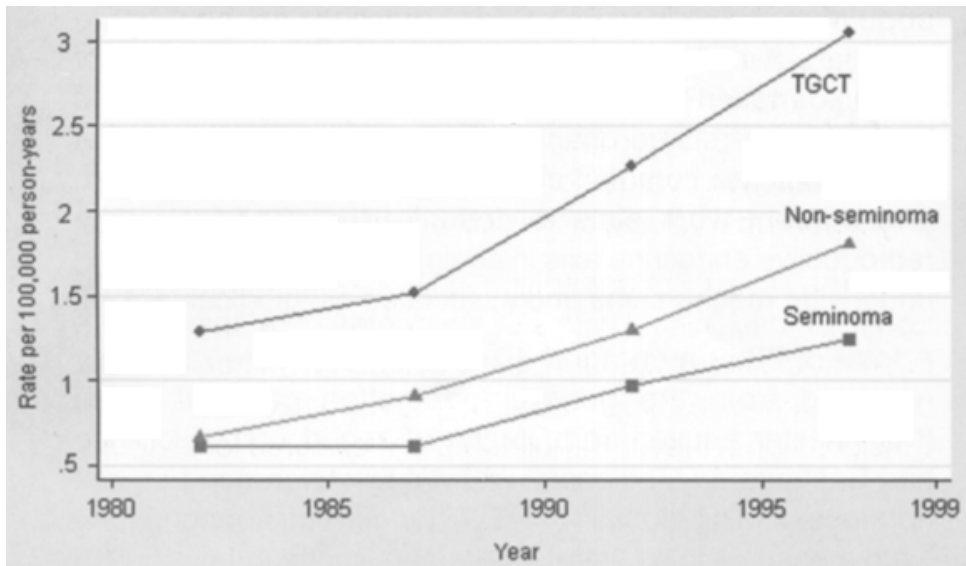

Age standardised (word population) incidence rates of testicular germ cell tumors (TGCTs) from 1980-1984 to 1995-1999 in Southern France.

Results : Over the whole of the study period, we observed a marked increase in the incidence of testicular cancer (cf. figure), for both seminomas and non-seminatous germ-cell tumours (mean annual increase $5 \%$ and $8 \%$, respectively). The incidence rate rose from 1.27 to 3.04 for 100000 men between 1980 and 1999. The age-cohort submodel appeared to be predictive of the time trend of testicular cancer incidence and revealed the existence of a birth-cohort effect.

Conclusion : The increased incidence of testicular cancer recorded in the Midi-Pyrenees region over the last 20 years reveals a birth-cohort effect similar to that observed in a number of European countries. This birth cohort effect is an argument in favour of the hypothesis now supported by numerous epidemiologists, that of environmental and/or occupational exposures to risk factors occurring early in life or even during the embryonic period.

$\underline{\text { OR } 11}$

Androgen receptor gene CAG repeat length as a modifier of the association between persistent organohalogen pollutant exposure and semen characteristics

\section{A. GIWERCMANa, L. RYLANDER ${ }^{b}$,} A. RIGNELL-HYDBOM ${ }^{b}$, B.A.G. JÖNSSON ${ }^{b}$, H.S. PEDERSENC, J.K. LUDWICKI ${ }^{f}$, V. LESOVOYg, V. ZVYEZDAYh, M. SPANO', G.C. MANICARDI', D. BIZZAROk, E.C. BONEFELD-JøRGENSENd,
G. TOFTe, J.P. BONDE', C. GIWERCMANa, T. TIIDOa, Y.L. GIWERCMANª AND INUENDO'

a Molecular Reproductive Medicine Research Unit, Department of Clinical Sciences, Fertility Centre, Malmö University Hospital, Lund University, Malmö ; $b$ Division of Occupational and Environmental Medicine and Psychiatric Epidemiology, Lund University, Lund

University Hospital, Lund, Sweden ;

c Centre for Arctic Environmental Medicine, Nuuk, Greenland;

d Institute of Public Health, Department of Occupational and Environmental Medicine, University of Aarhus, Aarhus, Denmark;

e Department of Occupational Medicine, Aarhus University

Hospital, Aarhus, Denmark;

f Department of Environmental Toxicology, National Institute of Hygiene, Warsaw, Poland ;

$g$ Regional Clinical Center of Urology and Nephrology,

Kharkiv State Medical University, Kharkiv, Ukraine;

$n$ Laboratory of Human Reproduction, Kharkiv State Medical University, Kharkiv, Ukraine;

i Section of Toxicology and Biomedical Sciences, BIOTEC-

MED, ENEA Casaccia Research Centre, Rome, Italy ; j University of Modena and Reggio Emilia, Reggio Emilia, Italy ;

k Istituto di Biologia e Genetica Universita' Politecnica delle Marche, Ancona, Italy ; I www.inuendo.dk.

Objectives : Exposure to Persistent Organohalogen Pollutants (POP) was suggested to impair male reproductive function. A gene-environment interaction has been proposed. No genes modifying the effect of POP on reproductive organs have yet been identified. We aimed to investigate whether the $C A G$ and GGN polymorphisms in the androgen receptor (AR) gene modify the effect of POP exposure on human sperm characteristics.

Design : Multi-centre prospective collection of biological material from partners of pregnant women (Greenland ; Kharkiv, Ukraine and Warsaw, Poland) and men from general population (Swedish fishermen).

Materials and Methods : Semen and blood from 680 men (mean (SD) age 34 (10) years) from Greenland, Sweden, Warsaw (Poland) and Kharkiv (Ukraine) were collected. POP exposure was assessed by measuring serum levels of 2,2', 4,4'5,5'-hexachlorobiphenyl (CB-153) and dichlorodiphenyl dichloroethene ( $p, p^{\prime}-D D E$ ). Semen characteristics (volume, sperm concentration, total count, proportion of progressively motile, morphology) and DNA Fragmentation Index (DFI). $C A G$ and $G G N$ repeat lengths were determined by direct sequencing of leukocyte DNA.

Results: Taking into consideration the inter-centre differences in exposure levels and genotype distribution and including age and time of abstinence as confounders, a statistically significant 
interaction was found between the CB-153 group and CAG repeat category in relation to sperm concentration and total sperm count $\left(p=0.03\right.$ and $p=0.01$, respectively). For $p, p^{\prime}-$ $D D E$, in the European cohorts a significant interaction was found in relation to DFI $(p=0.01)$. For $C A G<20$ sperm concentration and total sperm count were $35 \%$ and $42 \%$ lower, respectively, when the group with $\mathrm{CB}-153$ exposure above median was compared to that below the median. DFI was $40 \%$ higher in high $p, p^{\prime}$-DDE exposure group for $C A G \leq 21$.

Conclusions : The observed effect of POP exposure on sperm number was not seen when the genotype was not taken into consideration. This is the first study indicating geneenvironment interaction in relation to semen parameters. AR CAG repeat length might modify the susceptibility of an individual to the adverse effects of POP exposure on semen quality. These findings need to be confirmed by other studies.

Support : The study was supported by The European Commission to the 5th Framework Programme Quality of Life and Management of living resources, Key action four on environment and health, (Contract no. QLK4-CT-2001-00202), running 01.01.02-30.06.05. www.inuendo.dk. The Ukrainian part of the study was supported by a grant from INTAS (Contract no. 2001 2205). The Swedish part of the study has also been funded by the Swedish Medical Research Council (Grant No : 521-2004-6072) and the Swedish Research Council for Environment, Agricultural sciences and Spatial Planning.

OR 12

\begin{abstract}
Mechanisms underlying the age related fall in testosterone in men : results from the european male ageing study (EMAS)
\end{abstract}

\section{A. TAJAR1, F.C.W. WU1, T. O'NEILL1, J.D. FINN1, G. BARTFAI 2 , F. CASANUEVA 3 , G. FORTI4, A. GIWERCMAN5, I. HUHTANEIMI6, K. KULA7, M. PUNAB8, A.J.S. SILMAN1, D. VANDERSCHUEREN ${ }^{9}$ AND THE EMAS GROUP}

1 University of Manchester (UK), 2 Szeged University (Hungary), 3 Universidade de Santiago de Compostela

(Spain), 4 University of Florence (Italy), 5 Lund University

(Sweden), 6 University of Turku (Finland), 7 Medical

University of Lodz (Poland), 8 Medical University of Tartu

(Estonia), 9 Katholieke Universiteit Leuven (Belgium)

With the continuing rise in average life expectancy, care and prevention of disease/disability for the burgeoning elderly population have become priority targets for healthcare providers. A multi-centre longitudinal population cohort study was undertaken in Europe to investigate the relationships between age-related changes in hormones, their potential causes and the common clinical features of ageing in men. The present work aims to describe the changes in the reproductive endocrine axis in ageing men, and to identify the underlying mechanisms and associated aetiological factors.

A total of 3369 men aged 40 to 79 years were randomly recruited from the general population of eight cities (Manchester, Leuven, Tartu, Malmo, Szeged, Lodz, Florence and Santiago de Compostela). Total testosterone (TT), sex hormone binding globulin (SHBG), Luteinising hormone (LH) were measures in a central laboratory (Roche, Elecys E170) on a single fasting blood sample obtained before $11.00 \mathrm{~h}$. Free $T(F T)$ was calculated from TT and SHBG.

There was a significant age-related decline in TT and FT and an increase in LH and SHBG.

Multiple linear regression, linear spline fitting and multinomial regression analyses were used to investigate the combined effects of age and other predictors. We identified four distinct loci of dysregulation in the feedforward/feedback mechanism in the reproductive endocrine axis, which simultaneously contributed to the overall pattern of circulating hormone changes with increasing age. Each of these mechanisms was associated with a specific factor/aetiology.

Age : with increasing age, the testicular response to $\mathrm{LH}$ was impaired and compensated by increased LH The testicular dysfunction was amplified by the age-related increase in SHBG which resulted in a steeper decline in FT compared to TT.

Obesity : (general and central) - high BMI and waist circumference were associated with low TT and FT. This was not accompanied by a compensatory rise in $\mathrm{LH}$ indicating the presence of hypothalamus/pituitary dysfunction.

Co-morbidity : was associated with a more exaggerated rise in $\mathrm{LH}$ in men over the age of 70 indicating that testosterone feedback at hypothalamus/pituitary level was impaired or attenuated.

Smoking : increased SHBG, total testosterone and LH with only a minimal change in free $T$. This was compatible with a primary effect on the liver which triggers the initial rise in SHBG followed by a resetting of the feedback at a higher level of LH to maintain normal free testosterone.

We conclude that the age-related decline in circulating testosterone embraces a combination of specific dysregulations of hypothalamic-pituitary-testicular axis functions, each of which is associated with a distinct risk predictor. These mechanisms suggest that ageing is associated with compensated primary hypogonadism which develops into overt primary hypogonadism in the presence of comorbidity. Obesity on the other hand is associated with secondary (hypogonadotrophic) hypogonadism. Smoking is associated with increased TT due to elevated SHBG.

email : abdelouahid.tajar@manchester.ac.uk. 


\section{Analysis of $X-Y$ interactions in developing spermatids and characterisation of novel $Y$ transcripts}

\author{
L. FERGUSON1, P.J.I. ELLIS1, P.S. BURGOYNE2, \\ N.A. AFFARA 1 \\ 1 University of Cambridge Department of Pathology, \\ England \\ 2 National Institute for Medical Research, England \\ If268@cam.ac.uk
}

Other deregulated transcripts indicate downstream responses to the deletions. The array data highlighted the spermatidspecific up-regulation of X-linked genes in these deleted models (2).

Figure 2 : shows the $X$ chromosome bias of up-regulated genes in the $100 \%$ Yq deletion model.

Southern blotting and BLAST searches were used to determine autosomal, $X$ or $Y$ linkage for novel unmapped transcripts, and to confirm the mapping of selected deregulated transcripts. Intriguingly, we discovered that an up-regulated transcript, the RIKEN CDNAAK006152, had a male specific locus (figure 3a). This transcript was further mapped to Yp by PCR on genomic DNA from three mouse lines with deletions on $Y p$, indicating this gene is located either on telomeric Yp or within the centromeric Rbmy cluster (figure 3b). Transcription of the male specific locus AK006152 was shown by RT-PCR (figure 3c) to be testis specific.

Figures : $3 a$ : Southern blot identifying male specific band. $M$ Objective : A series of mouse lines with deletions on the $Y$ chromosome display phenotypes ranging from teratozoospermia to complete infertility and grossly abnormal sperm heads, depending on the extent and location of the deletion. Some of the sub-fertile models display a sex ratio distortion towards females. We have extended our transcriptional analysis of these models to a comprehensive gene set and mapped a selection of genes whose expression is altered by the deletions. Our goal is to identify novel $Y$ linked genes contained within the deletions and elucidate downstream genetic mechanisms linking genotype to phenotype.

Design / Materials and Methods : Array and quantitative RTPCR data $(1,2)$ was extended to wider gene-sets (a 36,000 probe oligonucleotide set and the MmcDNAv1 chip.) Labelled testis CDNA from each of four $Y$ deletion models was cohybridised with labelled testis cDNA from wild-type mice of the same strain as a common control. Lowess normalisation and Z-score analysis was used to select significantly regulated genes. Expression profiles were confirmed using RT-PCR. The chromosomal location and copy number of novel (previously uncharacterised) transcripts of interest was determined via Southern blotting and confirmed by PCR analysis of genomic DNA from the deleted models.

Figure 1 : MA plots of oligonucleotide arrays for each $Y$ deletion model. Red plots are significantly up or down regulated transcripts with a Z score $>1.28(p=0.1)$.

Figure 2 : Genomic distribution of significantly up-regulated genes in the $100 \% \mathrm{Yq}$ deletion model with respect to chromosomal gene density (ensembl) is biased towards the $X$ chromosome.

Results : Figure 1 : shows the significant differentially expressed transcripts (red) in the deletion models. Downregulated $Y$-linked transcripts indicate loci deleted from the $Y$ chromosome in the various models. Two down-regulated transcripts with genomic loci within the Sly (Sycp3 like $Y$ linked) gene family were identified, one of which is transcribed in anti-sense with respect to Sly.

$=$ male, $F=$ female.

$3 b$ : Genomic PCR identifying the presence of the loci in all 3 deletion models indicating a distal or proximal location on Yp common to all 3 models.

$3 c: R T-P C R$ confirming expression of the transcript is testis specific.

Southern blotting has also identified $Y$ chromosome homologues of other up-regulated $X$-linked and autosomal genes (data not shown).

Characterisation of deleted Yq linked genes may help in understanding the mechanism linking these deletions to the up-regulation of $X$-linked and Yp-linked genes and may be important in defining the phenotypes of these $Y$ deletion models. (3)

Conclusions : We can conclude that while there is an overall deregulation across the genome in the mouse $Y$ chromosome deletion models, the number of genes up regulated on the $X$ chromosome is significantly above average. This indicates a de-repression of $X$-linked genes (and some Yp-linked) as a consequence of these MSYq chromosomal deletions.

We also conclude that several significantly down-regulated, uncharacterised transcripts in the $\mathrm{Yq}$ deletion models have $\mathrm{Yq}$ genomic loci and may have a role in the phenotypes of these models.

Further studies of these novel Yq transcripts and the upregulated $X$ and $Y q$ linked genes may provide insight into the mechanism of this proposed competitive interaction of the $X$ and $Y$ chromosomes in murine spermatogenesis.

\section{Support :}

1. Ellis P.J.I. et al. Hum. Mol. Gen., 2005, 14, 2705-2715.

2. Ellis P.J.I. et al. Mol. Hum. Reprod., 2004, 10, 271-281.

3. Toure A., E.J. Clemente et al. Genome Biol., 2005, 6, 102.0102.15 


\section{Germline hMLH3 variants in maturation arrest}

\author{
C. FERRAS1, X. ZHOU2, S. FERNANDES1, \\ M. SOUSA1-3, A. LINDBLOM2, A. BARROS1-4
}

1 Genetics Department, Faculty of Medicine, University of Porto, Portugal ; 2 Department of Molecular Medicine, Karolinska Institutet, Stockolm, Sweden ; 3 Lab Cell Biology, ICBAS, University of Porto, Portugal ; 4 Centre for Reproductive Genetics A Barros, Portugal. C. Ferras (HYPERLINK "mailto:cferras@med.up.pt)1" cferras@med.up.pt)

Objective : To investigate whether $\mathrm{hMLH} 3$ gene mutations could be associated with meiosis impairment and consequently with human maturation arrest.

Design : MutL homologue 3 is a member of a family of conserved proteins involved in DNA mismatch repair during replication and meiotic recombination. MLH3-deficient mice do not show microsatellite instability (MSI) and are sterile due to meiotic arrest (1). Hereditary nonpolyposis colorectal cancer (HNPCC) is commonly associated with defects of DNA MMR genes and MSI, but involvement of the human MLH3 gene in DNA MMR is currently under debate, as mutations have either been associated or found to be absent in HNPCC. However, there is no experimental evidence for a similar hMLH3 involvement in human meiosis.

Material and Methods : Thirteen spermatogenic arrest patients studied for $A Z F / D A Z$ deletions were used for $\mathrm{MLH} 3$ mutation analysis. Genomic DNA was isolated from the testicular biopsies using a DNeasy Tissue Kit (Qiagen). MSI status was determined using the HNPCC MIS test (Roche) and the BAT34 mononucleotide marker. The 12 coding exons and exon-intron boundaries of the $\mathrm{hMLH} 3$ gene were studied by DHPLC and sequencing $(2,3)$. The primary spermatocyte stage arrest on the testicular biopsies was confirmed by FISH analysis of chromosomes $7 / 18 / \mathrm{X} / \mathrm{Y}$ in isolated germ cells (4). Primary MA was diagnosed when no evident cause for the meiotic arrest was found. Secondary MA was used as a control group and was established with patients with conserved spermatogenesis in their past history, AZFC and DAZ1/DAZ2 microdeletions (5) or hormonal disturbs like hypogonadotrophic hypogonadism.

Statistics analyses were performed by using the difference between two proportions test (Statistica, vs. 5.1) with a probability value of 0.05 being regarded as significant.

Results : All MA patients had a normal karyotype $(46, X Y)$ and none presented a personal or family history of HNPCC. Four out of 13 MA patients were considered primary MA and the remaining 9 cases secondary MA. MSI was negative for all patients. T2896C was detected in one secondary MA. C2531T combined with IVS9+66G->A were identified only in primary MA cases ( 3 of 4 patients, $75 \%$; $95 \% \mathrm{Cl} 21.9-98.7 \%$; $p=0.003$ to secondary MA cases. Secondary MA cases revealed none of these changes ( 0 of $9,0 \% ; 95 \% \mathrm{Cl} 0-37.1 \%)$. The intronic variant IVS9+66G->A was identified in 5 of secondary MA cases $(55.6 \%, 95 \% \mathrm{Cl} 22.7 \%-84.7 \%)$ : two with past history of spermiogenesis, two with DAZ1/DAZ2 microdeletions and one with panhypopituitarism.

Conclusions : Our results suggest that primary spematogenic arrest is associated with two simultaneous (C2531T and IVS9+66G/A) $h M L H 3$ variants. As isolated C2531T and IVS9+66G/A variants were previously described in several families with colorectal cancer as well as in controls, the presence of two simultaneous $\mathrm{hMLH} 3$ variants in patients with primary MA might represent an additive predisposing risk and be the cause of spermatogenic arrest.

Support :

1. Lipkin et al. (2002),

2. Liu et al. (2003),

3. Loukola et al. (2000),

4. Sousa et al. (2002),

5. Ferrás et al. (2004).

$\underline{\text { OR } 15}$

\section{Administration of estradiol stimulates proliferation of premieiotic germ cells and facilitates precocious maturation of Sertoli cells, promoting germ cell survival}

\section{R. WALCZAK-JEDRZEJOWSKA, J. SLOWIKOWKA-HILCZER, K. MARCHLEWSKA, K. KULA}

\author{
Department of Andrology \& Reproductive Endocrinology, \\ Medical University of Lodz, Poland. \\ E-mail:kkula@csk.umed.lodz.pl
}

Estradiol may participate in spermatogenic onset but its roles in Sertoli and germ cell multiplication and apoptosis are not clear.

Methods : Newborn male rats were daily injected between the 5th and 15th day of life with: $12.5 \mu \mathrm{g}$ of estradiol benzoate (EB) or $7.5 \mathrm{IU}$ of human purified FSH (hFSH) or EB+hFSH or solvents (control-C). On the 16th day blood was taken for hormone measurements and one testis for histology. Proliferation of germ and Sertoli cells were quantitatively evaluated by incidence of PCNA positive $(+)$ and apoptosis by TUNEL+ cells. 
Results : I) Blood level of testosterone was reduced to $25 \%$ of $C(p<0.01)$ after $E B$, to $49 \%$ of $C(p<0.01)$ after $E B+h F S H$ and increased to $186 \%$ of $C(p<0.05)$ after hFSH. Blood concentrations of prolactin increased to $1425 \%$ or $1265 \%$ of $\mathrm{C}$ after $\mathrm{EB}$ or $\mathrm{EB}+\mathrm{hFSH}$. $(\mathrm{p}<0.001)$. Blood triiodothyronine was slightly reduced by EB $(84 \%$ of $C, p<0.05)$ and by $h F S H$ $(90 \%$ of $C, p<0.05)$ and not changed after $E B+h F S H$. EB did not influence blood levels of FSH or LH.

II) In all groups first spermatogenesis progressed up to pachytene spermatocytes. Testes weight and tubule length were reduced by half of $C$ after $E B(p<0.001)$ and were doubled after hFSH or EB+hFSH $(p<0.001)$.

Seminal tubule diameter was reduced by EB $(69.5 \pm 2.6 \mu \mathrm{m}$ vs. $79.0 \pm 2.8$ in $C, p<0.001$ ), was stimulated by $E B+h F S H$ $(94.1 \pm 1.9 \mu \mathrm{m}, \mathrm{p}<0.001)$ and not changed by hFSH alone. The same concerned mean area of Sertoli cell nucleus. Similarly, while the formation of seminal tubules lumen increased by $3150 \%$ of $C(p<0.01)$ after $E B+h F S H$, it was not affected by hFSH or EB alone, indicating that precocious completion of Sertoli cell maturation appeared after combination of estradiol and $\mathrm{FSH}$ with a possible involvement of prolactin.

III) Incidence of PCNA+ germ cells was increased by all treatments: $90.8 \pm 3.7 \%$ of cells for $\mathrm{EB} ; 92.5 \pm 2.1$ for $\mathrm{hFSH}$ and $91.2 \pm 1.6$ for $E B+h F S H$ vs. $77.7 \pm 2.2$ for $C(p<0.001)$. Incidence of TUNEL+ germ cells was stimulated by EB to $196 \%$ of $C(p<0.05)$, not affected by $\mathrm{hFSH}$, and inhibited to $32 \%$ of $\mathrm{C}(\mathrm{p}<0.05)$ after $\mathrm{EB}+\mathrm{hFSH}$.

IV) Incidence of Sertoli PCNA+ cells was increased after hFSH to $420 \%$ of $C(p<0.001)$ and reduced after EB or $\mathrm{EB}+\mathrm{hFSH}$ to $25 \%$ of $\mathrm{C}(\mathrm{p}<0.01)$. Incidence of Sertoli TUNEL+ cells was increased after EB to $967 \%$ of $C(p<0.05)$ and not affected by $\mathrm{hFSH}$ or $\mathrm{EB}+\mathrm{hFSH}$.

\section{Conclusions :}

1) Administration of estradiol decreases testis maturation and secretion of testosterone but increases secretion of prolactin.

2) Estradiol, FSH and possibly prolactin increase proliferation of germ cells and hence all hormones may be important for triggering spermatogenesis.

3) Estradiol in synergism with $\mathrm{FSH}$ and possibly prolactin (but neither one of the hormone alone) induce acceleration of testis growth and Sertoli cell maturation, what in turn protects germ cells from apoptosis.

4) While FSH stimulates, administration of estradiol inhibits proliferation of Sertoli cells facilitating the shift into nonproliferative functional phase of Sertoli cell development.

\section{Transition protein (TP) expression in spermatids detected in ejaculates from Infertile men}

\section{Y. SOFFER, S. BECKER, L. YOGEV ${ }^{\star}$, L. SHOCHAT, L. LEWIN, R. GOLAN}

Department of Human Molecular Genetics and Biochemistry Sackler School of Medicine, and *Institute for the Study of Fertility, Tel Aviv Sourasky Medical Center. Tel-Aviv University.

Objective : TPs are DNA binding nuclear proteins found in spermatids during their differentiation. In rats, mice and humans TP2 appears first, shortly followed by TP1. Then protamine appears. The objective was to detect TP expression in seminal spermatids from infertile men.

Design : Out of 60 human semen samples from infertile men submitted to flow cytometry (FC) using propidium iodide staining, 23 samples demonstrated immature haploid cells on FC histograms, with noncondensed immature 1c DNA peaks. These samples were submitted to immunofluoresent staining of TP1 and TP2 and examined under confocal microscopy.

Materials and Methods : Samples were smeared on slides covered with polylysine and incubated in citrate buffer $(0.1$ M) at $900 \mathrm{C}$ for 10 minutes. Antibodies against transition proteins TP1 and TP2 (provided by Dr. W. S. Kistler) were added and incubated overnight, slides washed ( 3 times in Triton $\mathrm{x}-1000.1 \%$ ) and secondary antibody conjugated with FITC) was added for fluorescence and confocal microscopy. Sequential optical sections were prepared to demonstrate that TP expression was found inside the nuclei.

Results : TP expression was observed in 12 samples out of 23. In 9 samples, TP1 \& TP2 were both expressed and in 3 other samples, one of them only. Sequential optical sections using confocal microscopy demonstrated TP expression inside the nuclei. Flow cytometry performed on the semen samples revealed 4 peaks of haploid immature cells with various degrees of condensation. No clear relationship was found between degree of condensation and appearance of TP in the spermatids.

Conclusion : TP expression was demonstrated inside the nuclei of spermatids in about half of samples of infertile human semen samples containing immature haploid cells indicating disturbance of differentiation in spermatids. Similarities between sperm defects found in TP's mutants animals and infertile patients (Shirley et al., 2006) highlight the importance of TP's expression research in infertility. In this study, the heterogeneity of our infertile patients group and its multifactorial etiology didn't allow us to correlate TP's expression with clinical data.

Support : In part, by a grant of Israel Ministry of Health Chief Scientist Office. 
$\beta$ as independent parameters and FNA biopsy result as the dependent parameter revealed that only Inh- $\beta$ was a significant predictor of the FNA result $(r 2=0.289, p<0,001)$.

\section{The diagnostic role of Inhibin- $\beta$ in subfertile men and its correlation with testicular cytology}

\author{
P. POLICHRONOU, TH. MIKOS, D.G. GOULIS, \\ G. GRIMBIZIS, A. PAPANICOLAOU, S. GEROU, \\ J. BONTIS, J. PAPADIMAS
}

Unit of Reproductive Endocrinology, 1st Department of Obstetrics \& Gynaecology, Aristotle University of Thessaloniki, Greece
Conclusions : Basal serum Inh- $\beta$ seems to constitute an important diagnostic parameter in male subfertility, as it reflects both Sertoli cell reserve and testicular cytology. On the other hand, Sertoli cell reserve estimation, as estimated by EFSERT, does not seem to provide additional information for the diagnostic or the therapeutic approach of the subfertile male.

Presenting author: Prof. J. Papadimas, 1st Department of Obstetrics \& Gynaecology, "Papageorgiou" General Hospital, Periferiaki Odos, Nea Efkarpia, 564 03, Thessaloniki, Greece.

OR 18

Testosterone undecanoate is detected in seminal
plasma and significantly increases serum
dihydrotestosterone

\section{G. MITIOS, N. KAPOLLA, H. LASS*, E. KOUKKOU,} S.C. NICOPOULOU, D.A. ADAMOPOULOS

Objective : Inhibin- $\beta$ (Inh- $\beta)$ is produced almost exclusively by Sertoli cells and controls FSH secretion through a negative feedback mechanism. The role of Inh- $\beta$ as a diagnostic tool in male infertility is not fully elucidated. The aim of this study was to determine basal and stimulated Inh- $\beta$ serum levels in fertile and infertile men and to correlate them with testicular cytology.

Design : Prospective, cross-sectional, clinical study.

Materials and Methods : Sixty-seven subfertile and 29 fertile men underwent clinical, sperm, basal hormonal and dynamic Sertoli-cell evaluation (EFSER Test: serum Inh- $\beta$ levels before and $24 \mathrm{~h}$ and $48 \mathrm{~h}$ after administration of $300 \mathrm{IU}$ rhFSH subcutaneously). Genetic studies were applied as appropriate. Fifty-four of the subfertile men underwent testicular Fine Needle Aspiration biopsy (FNA). The final clinical diagnoses were: Idiopathic Non-Obstructive Azoospermia (INOA - $n=$ $31,46 \%, 5$ of them with Late-Onset Hypogonadism - LOH), varicocele $(n=14,21 \%)$, cryptorchidism $(n=10,15 \%)$ and other causes $(n=12,18 \%)$.

Results : There was significant difference between subfertile and fertile men regarding the basal (55.8 \pm 51.2 vs $115.7 \pm$ $54.0 \mathrm{pg} / \mathrm{ml}$, respectively, $\mathrm{p}<0,001$ ) but not the stimulated Inh- $\beta$ levels $(64.6 \pm 71.8$ vs $69.7 \pm 23.6 \mathrm{pg} / \mathrm{ml}, \mathrm{p}=\mathrm{NS}$ at 24 $\mathrm{h}$ and $63.3 \pm 79.2$ vs $87.9 \pm 25.1 \mathrm{pg} / \mathrm{ml}, \mathrm{p}=\mathrm{NS}$ at $48 \mathrm{~h}$ ). Patients with INOA and LOH, INOA only and cryptorchidism had the most severe Sertoli cell damage according to basal Inh- $\beta$ levels followed by varicocele. There was significant correlation between basal Inh- $\beta$ levels and FNA findings ( $r=$ $0.488, p<0.01$ ) with lower Inh- $\beta$ levels corresponding to more severe cytology diagnoses (Sertoli Cell-Only Syndrome -SCOS and maturation arrest). We applied a Receiver Operative Characteristics (ROC) analysis for sperm retrieval in testicular FNA (i.e. cytological diagnoses of normal spermatogenesis and hypospermatogenesis). The area under the ROC curve was 0.663 for FSH and 0.725 for Inh- $\beta$, with a threshold level of $54 \mathrm{pg} / \mathrm{ml}$ having a sensitivity of $59 \%$ and a specificity of $86 \%$ for sperm retrieval. Multivariate stepwise logistic regression analysis having age, mean testicular volume, serum FSH, serum testosterone and basal serum Inh-

\author{
Endocrine Department, Elena Venizelou Hospital, \\ 11521 Athens \\ * Bioanalytics, NV, Organon Oss and Organon \\ Development GmbH, Waltrop, Germany
}

Objective : Testosterone undecanoate (TU) has been used as a complimentary treatment to tamoxifen citrate in men with idiopathic oligozoospermia (I.O.) with satisfactory results. It has been postulated that TU through a dihydrotestosterone (DHT) promoting effect improves mainly epididymal environment and consequently the quality of spermatozoa.

Design : To examine this possibility, TU has been administered (40 ng t.i.d.) to 18 healthy, normozoospermic volunteers for a week, with measurements of TU, total testosterone (TT), dihydrotestosterone (DHT), E2, SHBG, FSH, LH and PRL. Steroid hormones were measured in peripheral blood and on some occasions, in seminal plasma before and after TU administration. Moreover, steroid hormones were also measured in a small number of testicular tissue samples from 5 unrelated men who also received TU before biopsy.

Materials-Methods : A home made assay was employed for TU estimations and AutoDelphia assays for the remaining hormones. Measurements in seminal fluid and testicular tissue were made following standard extraction techniques. Analysis of the results was made following standard procedures.

Results : TU had no effect on serum FSH (pre-: 6.0 0.7 , post-: $5.1 \pm 2.5 \mathrm{U} / \mathrm{mL}$ ), LH (pre-: $5.0 \pm 2.0$, post-: $4.0 \pm 2.0 \mathrm{U} / \mathrm{mL}$ ), 
PRL (pre-: $5.1 \pm 3.7$, post-: $4.0 \pm 2.0 \mathrm{U} / \mathrm{mL}$ ), SHBG (pre-: $30.0 \pm 13.8$, post-: $26.0 \pm 12.2 \mathrm{nmol} / \mathrm{L}$ ), TT (pre-: $4.88 \pm 1.63$, post-: $4.24 \pm 1.69 \mathrm{ng} / \mathrm{mL}$ ) and E2 (pre-: $27.6 \pm 8.0$, post-: $26.9 \pm 5.4$ $\mathrm{pg} / \mathrm{mL}$ ). Following TU treatment, a marked increase of DHT was observed in peripheral blood (pre-: $0.46 \pm 0.20$, post-: $1.14 \pm 0.74 \mathrm{ng} / \mathrm{mL}, P<0.001$, ratio: $0.41 \pm 0.15$, increase: $248 \%$ ) whereas no marked differences were noted for the remaining hormones. In seminal plasma, E2 and TT did not change after TU (E2: $73.4 \pm 19.3$ vs $70.2 \pm 24.3, T T: 0.6 \pm 0.1$ vs $0.7 \pm 0.2$ ) whereas DHT was not measured (by mistake). In the same fluid, TU was detectable in 6 of the 12 the samples assayed $(11.1 \pm 8.0 \mathrm{ng} / \mathrm{mL})$ and serum levels were comparable at $12.7 \pm 7.6 \mathrm{ng} / \mathrm{mL}$. The blood to seminal plasma ratio was 1.14 signifying that the average seminal TU concentration was $87.4 \%$ of that of peripheral blood.

In testicular tissue extracts, high $\mathrm{E} 2$ and $\mathrm{T}$ values (E2: $92.5 \pm 54.3 \mathrm{pg} / \mathrm{mL}, \mathrm{T}: 48.8 \pm 16.3 \mathrm{ng} / \mathrm{mL}$ of extract dilution) were seen but TU was undetectable.

Correlation analysis among the parameters examined showed that E2 and TU in serum and seminal plasma were significantly correlated both before and after treatment (pre: $\mathrm{P}<0.01,0.02$ post: $0.04,0.02)$. Moreover, correlations were noted before and on TU between E2 and DHT in serum $(P<0.02,0.01)$ and seminal plasma ( $P<0.001$ for both).

Conclusions : By and large, it appears that TU administration increased markedly serum DHT levels, making a beneficial effect on epididymal and accessory gland function a distinct possibility. TU was measurable in a number of seminal plasma samples in a concentration close to that of peripheral blood, indicating a clear presence of this lipophylic agent in the relevant compartments of the male genital tract. These two findings may contribute to the understanding of TU's beneficial effect in sperm dynamics in 1.O. patients.

Support : T and DHT hormone assays were performed at Department of Bioanalytics, NV Organon, Oss The Netherlands, whereas TU and other hormones were analyzed at the Department of Bioanalytics, Organon Waltrop $\mathrm{GmbH}$, Waltrop, Germany. The study was sponsored by NV Organon, Oss, The Netherlands.

\section{Characterizing the ampliconic composition of men with partial AZFc deletions - insights into how sequence rearrangement dynamics shape the AZFc locus}

\section{P. NAVARRO1,2, L. PEREIRA 3 , L. GUSMÃO3, J. CALHAZ4, C.E. PLANCHA2, J.GONÇALVES1}

1 Centro de Genética Humana, Instituto Nacional de Saúde Dr Ricardo Jorge ; 2 Unidade de Biologia da Reprodução, Instituto de Medicina Molecular, Faculdade de Medicina de Lisboa ; 3 Instituto de Patologia e

Imunologia Molecular da Universidade do Porto ; 4

Unidade Pluridisciplinar de Reprodução Humana, Hospital

de Santa Maria, Lisboa, Portugal

(joao.goncalves@insa.min-saude.pt)

Objective : The highly repetitive structure of the AZFc locus is a putative generator of $Y$ diversity. We hypothesize that such genetic variability could account for the variable phenotypes detected in patients with partial AZFc deletions. To test this, we characterized with novel amplicon-specific markers the exact structure of the AZFc locus of men with partial AZFC deletions, in order to identify sequence rearrangements that could be associated to specific infertility phenotypes.

Design : Gene and transcription unit copies previously mapped to AZFc amplicons were compared in silico in order to find amplicon specific nucleotide markers. Thus 7 novel ampliconspecific sequence family variants (SFVs) were designed and characterized in patients with partial AZFc deletions. These patients were selected after a previous STS screening of fertile and infertile men. Results were correlated to $Y$ haplogroup and (in)fertility phenotype.

Materials and Methods : The available reference sequence copies of the PRY gene, TTY4 transcript and the grey amplicons were aligned and compared (ClustalW software, EMBL-EBI). Significant amplicon-specific nucleotide variants associated to differential endonuclease restriction patterns were selected, resulting in 7 new amplicon-specific SFVs: b2, g1, gr, b3, g2, g3 and b4.

An initial screening for partial AZFc deletions was performed using a set of STSs (sY142, sY1197, sY1192, sY1291, sY1206 and SY1201) in 299 idiopathic infertile men and 220 fertile men. Deletions were confirmed by Eco-RV DNA blotting using the $49 f$ probe.

The AZFc ampliconic composition of the selected patients was characterized by sequencing all novel SFVS and by digestion profiling of previously described SFVs (DAZ-SNV I, DAZ-SNV II, sY586 and GOLY-SFV) and STS (Y-DAZ3).

Y-haplogroup analysis of the patients having deletions was performed by typing $35 \mathrm{Y}$-SNPs. Statistical analysis was performed using SPSS v13.0 software.

Results : Partial AZFc deletions were significantly more frequent in infertile men than in fertile controls : $16 / 299(5.4 \%)$ vs. $3 / 220(1.4 \% ; P<0.05)$. Although by STS typing all of the 19 deletions fit into the gr/gr profile, AZFC amplicon content in these patients varied significantly (14 different AZFC structures). Interestingly, the previously described gr/gr deletion mechanism could only be resolved in patients belonging to the $R$ haplogroup (12/19). In this haplogroup, $7 \mathrm{r} 1 / \mathrm{r} 3,3 \mathrm{~g} 1 / \mathrm{g} 3$ and $2 \mathrm{r} 1 / \mathrm{r} 4$ deletions were characterized, as well as 4 gene conversions and 3 amplicon inversions. No correlation between AZFc structure and fertility was detected since the ampliconic compositions recorded for fertile men were also observed in oligo and azoospermic patients.

Conclusions : The sequence rearrangement events operating 
in the AZFc locus result in high sequence diversity. This is observed on a large scale in different haplogroups, and on a smaller scale inside each haplogroup. This diversity results in different substrates for deletion mechanisms, which are reflected in the diverse products of the gr/gr deletion resolved by the new markers. Our data indicate that partial AZFC deletions reflect the evolutionary history of that particular AZFC locus and that, although a fertility risk, correlation between a specific AZFc composition and infertility cannot be established, suggesting that AZFC genes are part of a multifactorial network.

Support : Partially funded by Fundação para a Ciência e Tecnologia: \#SFRH/BD/16662/2004, and POCTI/ SAU/97/2001.

$\underline{\mathrm{OR}} 20$

\section{SNV (Single Nucleotide Variants) or STS (Sequence Tagged Sites) : Which choice for partial AZFc deletions and DAZ copies detection?}

\section{AKNIN-SEIFER1,2, R.L. TOURAINE2, A.K. FAURE3, K. MCELREAVEY 4 , J. CHOUTEAU 5 , R. LEVY1}

1 Laboratoire de Biologie de la Reproduction, Hôpital Nord, CHU de Saint-Etienne. 2 Laboratoire de Génétique de I'hopital Nord, Saint-Etienne. 3 Laboratoire de Biologie de la Procréation, $\mathrm{CHU}$ de Grenoble. 4 Reproduction, Fertilité et Populations, Institut Pasteur, Paris. 5 Clinilab, SaintMartin d'Hères. Tel. : 04-77-82-83-07 Fax : 04-77-8287-84e-mail: rachel.levy@chu-st-etienne.fr

Aim of study : Partial deletions of the AZFc region (AZoospermia Factor) of the $Y$ chromosome have been reported to be a significant risk factor for oligo-/azoospermia. Within the AZFc region, the DAZ (Deleted in AZoospermia) gene family is organized into two clusters with a variable number of copies with $99.9 \%$ homology. DAZ1/DAZ2 deletions have been found only in infertile men, as opposed to DAZ3/DAZ4 deletions found also in fertile men. The aim of the present study is to compare two methods for partial AZFC deletions detection: one based on STS (Sequence Tagged Sites) and the other one based on SNV (Single Nucleotide Variant) which allows to distinguish one copy from the 3 others.

Design and Location : In this retrospective study, first, we used previously published STS to screen infertile patients and controls in order to detect partial deletions of AZFC region. Secondly, we designed a method to detect SNV using DHPLC
(Denaturing High Performance Liquid Chromatography).

Materials and Methods : Patients : 850 infertile patients exhibiting a non-obstructive oligozoospermia or azoospermia and 72 normozoospermic fertile men have been analyzed. SNV: Detection of SNV-I, SNV-II, SNV-III (for DAZ4, DAZ1 and DAZ2 respectively) using DHPLC. DAZ3 is identified using a gene copy specific STS amplification. STS : Screening of 6 STS: sY1161, sY1197, sY1191, sY1291, sY1206 and sY1201, in biplex.

Results : STS : grigr deletions (corresponding to the absence of sY1291), b1/b3 deletions and b2/b3 deletions were found respectively in 38 men (4.8\%), 2 men (0.3\%) and 2 men $(0.3 \%)$ with oligozoospermia or azoospermia. They were not detected in fertile normozoospermic men. SNV: $2.9 \%$ of patients and $0.5 \%$ of controls exhibited a DAZ1 deletion. $4.9 \%$ of patients (38) but none normospermic exhibited a DAZ4 deletion. Surprisingly, DAZ2 and DAZ 3 "deletions" could be found both in controls and in patients $(23 \%$ versus $14.8 \%$, and $10 \%$ versus $16 \%$, respectively).

Concordant results between the two methods were observed for $33 \mathrm{gr} / \mathrm{gr}$ deletions: 17 were DAZ1/2 deletions (57\%) and 16 were DAZ3/4 deletions ( $43 \%$ ). The 5 others cases were $\mathrm{gr} / \mathrm{gr}$ deletions according to STS with unexpected results with SNV analysis: one DAZ1/DAZ2/DAZ4 deletion, two with an isolated DAZ2 deletion, and two without any DAZ copy deletion. We also confirmed the two $b 1 / b 3$ and the two $b 2 / b 3$ deletions using SNV analysis. We consider that the SNV-III and YDAZ3 used for DAZ2 and DAZ3 are not useful, since they are absent in control patients (either these markers are not always present, or the absence of the corresponding copy has no consequence on fertility). Conversely, the absence of DAZ1 and DAZ4, detected using SNV-II and SNV-I respectively, is predictive of infertility and correlates well to the STS detection results (exact Fisher's test $p<10-3$ ). No correlation with semen or clinical parameters could be found for infertile patients, neither with STS or SNV results.

Conclusions : Study of SNV II and I, present in DAZ1 and DAZ4 respectively, by DHPLC, seems to be an easy method, completing the STS analysis. SNV analysis can bring additional information on the size and type of the partial AZFc deletions. Together with the $Y$ haplogroups determination (in progress), STS and SNV combined analysis is needed to have a more accurate prognostic tool for these small rearrangements of the Y chromosome.

\section{References :}

Fernandes A.T., Fernandes S., Goncalves R. et al. : DAZ gene copies : evidence of $Y$ chromosome evolution. Mol. Hum. Reprod., 2006 Jun 15.

Repping S., Van Daalen S.K., Brown L.G. et al. : High mutation rates have driven extensive structural polymorphism among human $Y$ chromosomes. Nat. Genet., 2006, 38 : 463-467.

Lynch M., Cram D.S., Reilly A. et al. : The Y chromosome gr/gr subdeletion is associated with male infertility. Mol. Hum. Reprod., 2005, 11 : 507-512.

Lin Y.W., Thi D.A., Kuo P.L. et al. : Polymorphisms associated 
with the DAZ genes on the human $Y$ chromosome. Genomics, $2005,86: 431-438$.

Ferlin A., Tessari A., Ganz F. et al. : Association of partial $A Z F c$ region deletions with spermatogenic impairment and male infertility. J. Med. Genet., 2005, 42 : 209-213.

Giachini C., Guarducci E., Longepied G. et al. : The gr/gr deletion(s) : a new genetic test in male infertility ? J. Med. Genet., 2005, 42 : 497-502.

OR 21

\section{Natural transmission of USP9Y gene mutations : a new perspective on the role of AZFa genes in male fertility}

\author{
C. KRAUSZ, S. DEGL'INNOCENTI, F. NUTI, A. MORELLI, \\ E. GUARDUCCI, G. FORTI \\ Department of Clinical Physiopathology, Andrology Unit, \\ University of Florence, Italy Corresponding \\ author:c.krausz@dfc.unifi.it
}

Objective : Deletions of the AZoospermia Factor Regions (AZF) of the $Y$ chromosome are associated with severe spermatogenic failure and represent the most frequent molecular genetic cause of azoospermia and severe oligozoospermia. The exact role of the candidate AZF genes is largely unknown due to both the extreme rarity of naturally occurring AZF gene specific mutations and the lack of functional assays. Only two cases of finely characterized and confirmed isolated AZF gene mutations have been reported to date. In both cases the mutations removed the USP9Y gene in the AZFa region. The associated phenotype in both cases was infertility due to severe impairment of the spermatogenesis.

Here, we report the fine characterization of two different deletions in the USP9Y gene (one of the two candidate genes in the AZFa region), which have been transmitted through natural conception in two unrelated families.

Materials and Methods : A total of 995 men affected by infertility have been screened for AZFa (USP9Y and DBY) and AZFb (elF1AY and HSFY) gene specific deletions in our laboratory. In addition, 350 normospermic men were screened for gene specific deletions and resulted not to be deleted for any of the two USP9Y markers.

Since USP9Y is composed of 46 exons, we use two primer pairs, one mapping to exon 22 (GeneBank G54725), and another to exon 46 (GeneBank G34983). We used a number of additional gene specific markers to localize the breakpoints in patient two patients. The deletion junction has been then amplified using long range PCR, and the amplified products was sequenced by automatic sequencer (ABI PRISM 310, Applied Biosystems).

Results : After the routine and gene specific screening we found two patients (1115 and A333) with partial USP9Y deletion (one of the two candidate genes in the AZFa region). The deletion in patient $A 333$ removed the first 28 exons, whereas in patient 1115 the last 13 exons of the USP9Y gene. In patient 1115, RT-PCR revealed the presence of the 5 ' end of USP9Y transcript and, as expected, the absence of its 3 ' end, suggesting the translation of a truncated protein. In patient A333 it is highly unlikely that the undeleted, 3' portion, of USP9Y was transcribed, as the deletion removed $28 \mathrm{~kb}$ of sequence $5^{\prime}$ of the normal position of the first exon. The screening of the male relatives of both subjects enabled us to detect the transmission of deletion through natural conception in both unrelated families. The associated mild testicular phenotype (moderate oligoasthenoteratozoospermia), in both cases, is in sharp contrast with that of the two previously reported infertile patients bearing a mutation of the same gene.

Conclusions : In conclusion, to date the USP9Y gene has been considered as one of the major Y-linked spermatogenesis genes, based on both its position within the AZFa region and previous reports that correlated USP9Y mutation to severe spermatogenic failure and infertility. This view is now substantially changed since our findings clearly demonstrate that during human spermatogenesis, USP9Y is more likely a fine tuner which improves efficiency rather than a provider of an essential function. More importantly, the observed natural conceptions suggest that the protein is not required for the final sperm maturation process or for the acquisition of sperm fertilizing ability providing a new perspective on the role played by the USP9Y gene in male fertility.

$\underline{\mathrm{OR}} 22$

\section{Effect of prostasomes on human spermatozoa. An in vitro study}

Objective : Prostate gland secretions are rich in prostasomes, vesicles known to play an important role in male fertility. Prostasomes have an unusually high concentration of cholesterol relative to phospholipids (2:1 a compared with 
In the laboratory, Picherit et al. have reported that human spermatozoa incubated for 3 hours with cyclodextrine (an acceptor of cholesterol) exhibited a strong correlation between the degree of tyrosine phosphorylation of a $107 \mathrm{KDa}$ protein and ionophore A23187-induced acrosome reactions.

It is known that loss of cholesterol from sperm membranes occurs during capacitation. Since evidence indicates that prostasomal molecules, especially cholesterol, can be transferred to sperm under certain conditions, we hypothesized that transfer of cholesterol to sperm membranes could affect some spermatozoa properties. Therefore, we investigated the effect of prostasomes on 1) the tyrosine phosphorylation of a $107 \mathrm{KDa}$ protein band, 2) sperm lipid profile.

Materials and Methods: Human spermatozoa were selected by Percoll density centrifugation and incubated for 3 hours at $370 \mathrm{C}$ under an atmosphere of $5 \% \mathrm{CO} 2$ in air, in the absence or presence of increasing concentrations of prostasomes. Quantities of prostasomes used for experiments were expressed in terms of cholesterol $(0.3 \mathrm{nmol}$ to $45 \mathrm{nmol}$ of cholesterol / $100 \mu$ l of incubation medium). After incubation either Western blotting analysis or HPTLC were performed.

Results : The cell viability measured after 3 hours incubation in the absence or presence of prostasomes was not modified compared with the sperm population viability measured before incubation.

We observed that tyrosine phosphorylation of the $107 \mathrm{KDa}$ protein band was inhibited when sperm were incubated with increasing concentrations of prostasomes; the differences relative to untreated controls were significant for 30 and 45 $\mathrm{nmol}$ of cholesterol per $100 \mu \mathrm{l}$ of incubation medium. We also noted a significant increase in the amount of cholesterol and the main classes of phospholipids. These enrichments were dose dependent.

Conclusion : We concluded that prostasomes in vitro are capable of inhibiting events (tyrosine phosphorylation) known to be activated during the capacitation process. The changes in the lipid composition after 3 hours incubation, and more specifically enrichment in cholesterol could be responsible of this inhibitory effect. These results suggest that prostasomes could in vitro maintain human sperm in a non capacitated state and partly explain the anti-capacitating properties of seminal plasma. Further in vitro studies are required to complement this work.

References : Picherit-Marchenay C., Bréchard S., Boucher D., Grizard G. : Correlation between tyrosine phosphorylation intensity of a 107KDa protein and A23187-induced acrosome reaction in human spermatozoa. Andrologia, 2004, 36 : 370377.

\section{Regulation of mitochondrial DNA content in human sperm: implications for male (in)fertility}

Human mitochondrial DNA (mtDNA) encodes 13 polypeptides of the electron transfer chain, the apparatus that is responsible for the generation of ATP through the process of oxidative phosphorylation. The integrity of the mitochondrial genome is particularly important in those cells with high energy demands, such as sperm. Its replication is dependent on nuclear-encoded factors, including DNA Polymerase Gamma (POLG) and mitochondrial transcription factor A (TFAM). POLG is the DNA polymerase specific to mtDNA replication. TFAM activates mtDNA transcription and thus replication, and is thought to be a regulator of mtDNA copy number. Down-regulation of TFAM was proposed to cause the 10-fold reduction in mtDNA copy number during spermiogenesis. Furthermore, low levels of expression of either of these two proteins have been associated with mtDNA-depletion syndromes.

The aim of this study was to investigate the significance of the expression of POLG and TFAM in the quality of human sperm and to determine whether these two factors influence sperm mtDNA content. We have analysed 93 sperm samples from men attending a Portuguese infertility clinic and categorized them into three groups, as evaluated according to the World Health Organization Guidelines (1999) : i) normozoospermic ; ii) samples with one or two abnormal semen parameters; and iii) oligoasthenoteratozoospermic (OAT). POLG and TFAM expression was evaluated using immunocytochemistry and Western Blotting. MtDNA content was determined in 42 samples by the ratio of mtDNA copies to the nuclear-encoded Beta-Globin gene, through real-time quantitative PCR. POLG and TFAM expression was localised to the midpiece of mature sperm.

The percentage of those sperm expressing these proteins was significantly lower in OAT than in normozoospermic samples $(P<0.01$ for $P O L G, P<0.05$ for TFAM). The mean $m$ tDNA copy number for human sperm was determined at 21.6 \pm 5.8 . However, in contrast to POLG and TFAM levels, the $\mathrm{mtDNA}$ content was significantly higher in OAT samples (46.7 $\pm 15.0)$ than in normal samples $(6.8 \pm 1.8, P<0.01)$ and in samples with 1 or 2 defects $(11.3 \pm 4.5, P<0.05)$. Indeed and paradoxically, a negative correlation was found between mtDNA copy number and the percentage of sperm expressing POLG $(R=-0.582, P<0.001)$. 
We propose that the reduction in mtDNA content observed in normal samples reflects normal spermiogenesis. The high number of mtDNA copies associated with OAT samples may reflect one of many outcomes arising from defective spermiogenesis. We further propose that, in normal samples, mature sperm increase their levels of POLG and TFAM expression in an attempt to compensate for the low mtDNA content. However, this mechanism appears to be ineffective due to the persistence of low copy number.

In conclusion, tight regulation of nucleo-mitochondrial interactions might be essential for the effective completion of spermiogenesis and subsequent sperm function.

A.A is supported by FCT Portugal (SFRH/BD/12665/2003). This work was supported by FCT (POCTI/ESP/38049/2001), by Instituto Investigação Interdisciplinar, University of Coimbra (III/BIO/50/2005) and by British Heart Foundation (PG/04/117).

\section{Tyrosine kinases and protein kinase A play different roles in capacitation and motility in human spermatozoa}

\section{LUCONI, G. VARANO, A. LOMBARDI, G. FORTI, E. BALDI}

Dept. Clinical Physiopathology Andrology Unit, University of Florence. Italy (e.baldi@dfc.unifi.it)

Although the role of protein phosphorylation/dephosphorylation in regulation of sperm functions is well known, the precise timing of activation and reciprocal function of protein kinase $A$ (PKA) and tyrosine kinases (TKs) in the development and maintenance of sperm motility and capacitation is still unclear. By western blot analysis of human sperm using PY20 antibody directed against tyrosine phosphorylated protein motif, we demonstrated that during sperm capacitation tyrosine phosphorylation is the first signal to be activated which is responsible of the time-dependent increase in sperm motility. Blocking TKs from the beginning of capacitation results in a significant inhibition of sperm motility parameters evaluated by CASA, associated by a reduction of PKA activity, evaluated using an antibody against PKA activated substrates. On the contrary, inhibition of PKA by H89 slightly affects sperm motility and tyrosine phosphorylation but only at the very first step of capacitation ( $30 \mathrm{~min}$ ), whereas such processes are unaffected at longer time ( $2 \mathrm{~h}-3 \mathrm{~h}$ ), suggesting a precise timing in regulation of sperm motility. In the model we propose, in fact, TKs are immediately activated when capacitation starts and act upstream of PKA, probably through tyrosine phosphorylation of AKAP3 which results in PKA recruitment and activation in sperm tails (Luconi et al, J Cell Sci, 2004). Among TKs activated during capacitation, p60src has been identified for the first time. Interestingly, PKA contributes to stimulate TKs, thus a positive loop is established between tyrosine phosphorylation and PKA activity. At longer time, the relative importance of PKA declines and motility seems to be mainly maintained by TKs, as demonstrated by the absence of effect of $\mathrm{H} 89$ at 2 hour capacitation. 